\title{
The Associations for Vaginal Point Doses of Vaginal Stenosis in Image-Guided Brachytherapy
}

\author{
Ekkasit Tharavichitkul ${ }^{1 *}$, Rungtip Jayavasti ${ }^{1}$, Razvan M. Galalae ${ }^{2}$, Damrongsak Tippanya1, \\ Sukon Prasitwattanaseree ${ }^{3}$, Wannapa Nobnop${ }^{1}$, Somvilai Chakrabandhu1, \\ Pitchayaponne Klunklin', Wimrak Onchan'1, Somsak Wanwilairat'1, Imjai Chitapanarux ${ }^{1}$ \\ ${ }^{1}$ The Division of Therapeutic Radiology and Oncology, Faculty of Medicine, Chiang Mai University, Chiang Mai, \\ Thailand \\ ${ }^{2}$ Faculty of Medicine, Christian-Albretch University, Kiel, Germany \\ ${ }^{3}$ Science and Technology Research Institute, Chiang Mai University, Chiang Mai, Thailand \\ Email: paan 31@hotmail.com
}

Received 2 June 2014; revised 1 July 2014; accepted 28 July 2014

Copyright (C) 2014 by authors and Scientific Research Publishing Inc.

This work is licensed under the Creative Commons Attribution International License (CC BY).

http://creativecommons.org/licenses/by/4.0/

(c) (i) Open Access

\begin{abstract}
Purpose: To evaluate the associations for vaginal dose points of vaginal stricture in image-guided brachytherapy. Materials and Methods: Twenty-six patients of locally advanced cervical cancer were treated with Image-Guided Brachytherapy (IGBT) with the dose at least 7 Gy per fraction to the D90 of High-Risk Clinical Target Volume (HR-CTV). The vaginal dose points of recommendations of the American Brachytherapy Society (ABS) were added into the plan and cumulative dose to these points was evaluated in Equivalent Dose of 2 Gy (EQD2) concepts. Results: The mean doses to right vaginal dose point (VR), left vaginal dose point (VL) and average dose of VR/VL ((VR + VL)/2) were 101.5 Gy, 98.2 Gy and 99.8 Gy in EQD2 concepts, respectively. Volume-based planning significantly reduced the cumulative dose in EQD2 concepts at vaginal points. At the median follow-up time of 22 months, grade- 2 vaginal stricture was observed in two patients. The incidences of vaginal stricture were not differed between the cumulative dose to vaginal dose points in EQD2 concepts of $\leq 90$ Gy versus $>90 \mathrm{~Gy}(P=1.000)$ and $\leq 100 \mathrm{~Gy}$ versus $>100 \mathrm{~Gy}(\mathrm{P}=0.815)$. Conclusion: No association for cumulative vaginal doses and events of vaginal stricture was found.
\end{abstract}

\section{Keywords}

Image-Guided Brachytherapy, Vaginal Dose Point, Vaginal Stricture, Associations

\footnotetext{
"Corresponding author.

How to cite this paper: Tharavichitkul, E., Jayavasti, R., Galalae, R.M., Tippanya, D., Prasitwattanaseree, S., Nobnop, W., Chakrabandhu, S., Klunklin, P., Onchan, W., Wanwilairat, S. and Chitapanarux, I. (2014) The Associations for Vaginal Point Doses of Vaginal Stenosis in Image-Guided Brachytherapy. Journal of Cancer Therapy, 5, 823-829. 


\section{Introduction}

Cervical cancer is the most common cancer in females in Thailand. In the year of 2005, the incidence of cervical cancer in northern Thailand was 23.5 per 100,000 [1].

In case of inoperable or locally advanced stages, radiotherapy (external beam radiotherapy plus brachytherapy) plays a major role in the theraputic management of this setting. Intracavitary brachytherapy for cervical cancer has been historically used for treatment based on x-ray concepts. According to the developments of ImageGuided Brachytherapy (IGBT) for the treatment of cervical cancer, many publications recently supported these novel techniques to improve the treatment results and betterments of bladder and rectum morbidities were reported extensively in these publications [2]-[5].

Vaginal toxicity, especially vaginal stricture, is the most common late toxicity that may cause the problem to quality of life. However, vaginal doses were not specifically addressed to most publications. To report vaginal dose burdens, vaginal dose points have been defined at the vaginal source level. Traditionally, the vagina has been defined as relatively radioresistant organs with low incidence of severe late radiation toxicity of only $1 \%$ 7\% [6] [7]. However, recent publications by Gondi et al. showed higher incidence of late vaginal toxicity in chemoradiation group [8]. Limited reported data onto vaginal effects of image-guided brachytherapy in volume and point approaches are available [9].

In our institute, image-guided brachytherapy was introduced and validated in specific research projects and showed promising interim outcome results and toxicity profiles [10]. However, vaginal toxicity was not reported so far. Thus, we performed this retrospective study to evaluate the effect of image-guided brachytherapy in vaginal dose points and toxicity profiles for patients treated by IGBT in our institute.

\section{Materials and Methods}

After approval from the ethics committee, patients who were treated with for radical radio-chemotherapy with IGBT for locally advanced cervical cancer in The Division of Therapeutic Radiology and Oncology, Faculty of Medicine, Chiang Mai University during January 2010-December 2011 were enrolled to evaluate the vaginal toxicity during the follow-up program. All patients were classified IIB-IIIB by FIGO clinical staging with upper one-third vaginal involvement, were between 18 - 70 years old and had good performance status. Patients with severe co-morbidity, emergency conditions (e.g. bleeding) that could not permit delays in treatment, pregnancy, history of previous irradiation or allergy were excluded from the study. Consent forms were signed by each patient prior to enrollment. Treatment schedules for these patients were shown as the followings.

\subsection{Whole Pelvic Radiotherapy (WPRT)}

All patients received EBRT of 45 Gy using Three-Dimensional Conformal Radiotherapy (3D-CRT) or Intensity-Modulated Radiation Therapy (IMRT). At the beginning, a Computed Tomography (CT) simulation was performed. The pelvic region from the L1-2 interspace to the whole vagina was scanned without intravenous contrast to obtain appropriate images of the patients in supine treatment positions and legs relaxed on the table. The slice thickness of CT scans was 5 mms without an inter-slice gap. After imaging processes was completed, Image data was sent to the Contouring and Treatment Planning. For the identification of Clinical Target Volume (CTV), the RTOG/JCOG recommendations were used in combinations of a guide to contourings [11]-[14]. The CTV was composed of cervix, uterus, adnexaes, the upper half of the vagina, and pelvic lymph nodes (common iliac Lymph Nodes [LNs], external iliac LNs, internal iliac LNs, obturator LNs and presacral LNs). Planning Target Volume (PTV) was defined by CTV plus $0.7-1.5 \mathrm{~cm}$ margin. Bladder, rectum, sigmoid colon, bowels and head of femurs were contoured as Organs At Risks (OARs). For CTV, the dose of 1.8 Gies per fraction, five fractions per week was prescribed to the total dose of $45 \mathrm{~Gy}$. The D98 (dose at $98 \%$ of volume) of CTV was calculated. For OARs, the doses at D2\% of the bladder, rectum and sigmoid colon were recorded for dose evaluation. During irradiation, patients were advised to preparing the bladder and rectum such as with CT simulations. Electronic Portal Imaging Devices (EPID) was used weekly to evaluate and correct for actual position. The CTV and OARs doses were calculated into equivalent dose in 2 Gy-fractions (EQD2) using the linear-quadratic model and assuming $\alpha / \beta$ ratio $=10$ for tumor and $\alpha / \beta$ ratio $=3$ for OARs [15].

\subsection{Concurrent Chemoradiation}

Concurrent chemoradiation with weekly cisplatin $40 \mathrm{mg} / \mathrm{m}^{2}$ for a maximum of five cycles was given to patients 
with an indication of combined radio-chemotherapy with sufficient kidney and bone marrow function. The dose of chemotherapy was modified according to a weekly assessment of creatinine clearance prior to each applied dose. Chemotherapy was interrupted when creatinine clearance was less than $40 \mathrm{ml} / \mathrm{min}$ and considered to be stopped when creatinine clearance was less than $30 \mathrm{ml} / \mathrm{min}$.

\subsection{Image-Guided Brachytherapy (IGBT)}

One-hundred and four applications in 26 patients of Image-Guided Brachytherapy (IGBT) with CT or Magnetic Resonance Imaging (MRI) were evaluated and 85.6\% (89/104 applications) of them were CT-based approaches. Tandem-ovoid or tandem-ring applicators were used for these patients. For MRI, GEC-ESTRO definitions were used to identify target volumes e.g. Gross Tumor Volume (GTV) or High-Risk Clinical Target Volume (HRCTV), and OARs [16]. Dose-volume histograms were calculated to consider the adequate dose to HR-CTV and limitations of OARs. In CT imaging, all HR-CTV and OARs were contoured according to the CT-standardized Contour Guidelines of Viswanathan et al. [17]. For the planning system, we used PLATO software (software BPS version 3.3.2 ${ }^{\circledR}$, Nucletron, an Elekta company, Elekta AB, Stockholm, Sweden) for the contouring and planning process. The prescribed dose started to $7 \mathrm{~Gy}$ per fraction to point A for all patients. Dose-Volume Histograms (DVHs) to HR-CTV and OARs were evaluated as point-based plan. Optimization was performed when the DVHs to HR-CTV and OARs did not correlate to GEC-ESTRO recommendations and aimed to keep the cumulative dose at $90 \%$ of HR-CTV to be at least 7 Gy per fraction. After optimization, the DVHs to HR-CTV and OARs were evaluated again as volume-based plan and patients were treated by optimized plan. The dose constraints on both volume-based to plan and point-based plan were recorded. For HR-CTV, the D90 (minimum dose covering $90 \%$ of target volume) of HR-CTV in all patients was recorded. For OARs, the D2cc (representing the minimum doses calculated at the most irradiated 2 cc volumes) of the bladder, rectum, and sigmoid colon were recorded. The HR-CTV and OARs total doses (WP-RT and IGBT) were calculated into equivalent doses in 2 Gy-fractions (EQD2) using the linear-quadratic model and assuming $\alpha / \beta$ ratio $=10$ for tumor and $\alpha / \beta$ $=3$ for OAR [15]. The EQD2 of WPRT and IGBT were added to evaluate the volume-based plan with regards to the DVH constraints corresponding to a prescribed dose of at least $80 \mathrm{~Gy}$ in EQD2 for tumor (D98\% of WP-IMRT plus D90\% of IGBT). For the OAR, total doses (D2\% of WP-IMRT plus D2cc of IGBT) constraints maintained $\leq 75$ Gy in EQD2 for rectum/sigmoid and $\leq 90$ Gy in EQD2 for the bladder, respectively [18].

\subsection{Vaginal Point Identifications}

To evaluate the vaginal doses, the two vaginal point doses on right and left sides (VR and VL) according to American Brachytherapy Society (ABS) guidelines were used to identify the vaginal dose points [19] (Figure 1).

The cumulative doses in Equivalent Dose of 2 Gy (EQD2) at vaginal dose points were calculated with the $\alpha / \beta$ of 3 for each patient in terms of point-based planning (point A prescription) and volume-based planning (D90 of HR-CTV). The vaginal-point doses were recorded in both sides of the vagina to be right and left vaginal-point

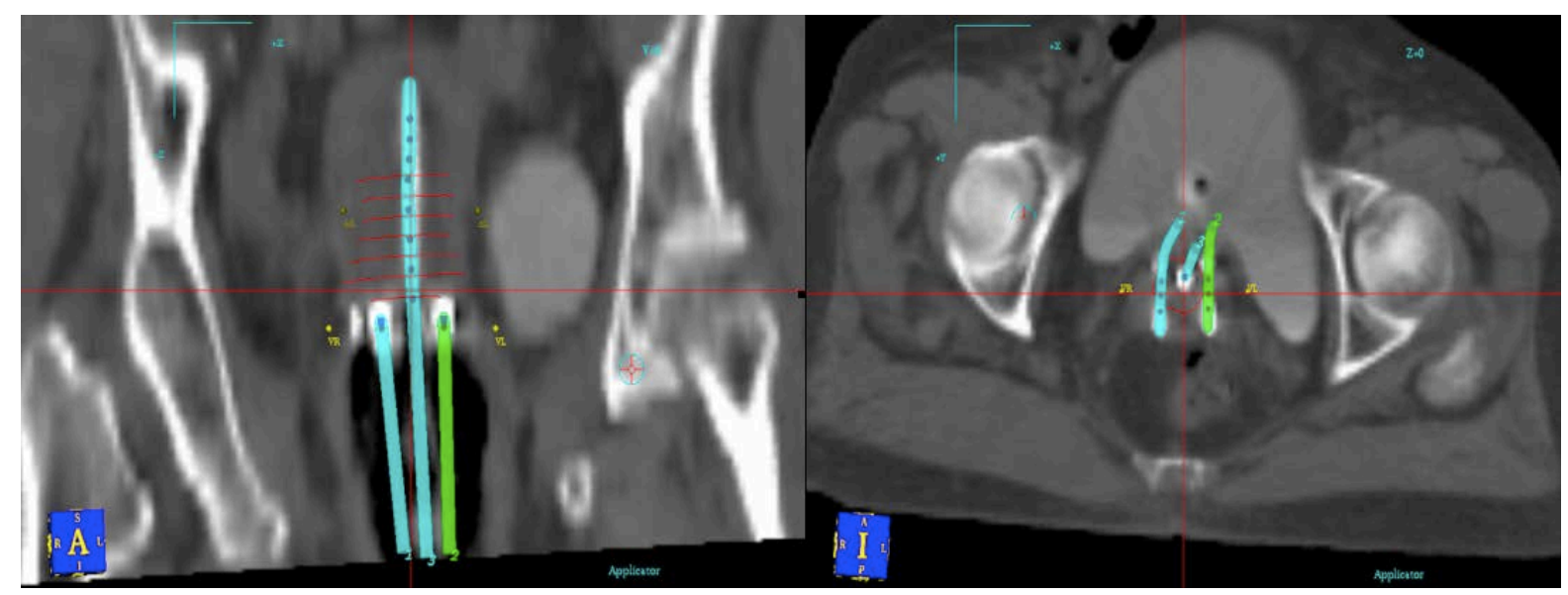

Figure 1. Coronal (left) and axial (right) views of CT-images showing the vaginal point doses (right (VR) and left (VL)) according to American Brachytherapy Society (ABS) guidelines. 
doses (VR and VL). The VR and VL dose for both volume-based plan and point-based plan were recorded to evaluate.

\subsection{Evaluation}

After the treatment was completed, patients were appointed to a vaginal examination (PV exam) in a follow-up program. Late complications from RT are defined as side effects developing 3 months after RT completion. For each endpoint, the grade in late morbidity will be the maximal grade ever recorded, even if the side effect later subsided. The data will be retrospectively collected from treatment data and patient record of each patient. Vaginal stricture was evaluated and recorded according to National Cancer Institute, USA; Common Terminology Criteria of Adverse Event (CTCAE) version 4.0.

\subsection{Statistical Analysis}

The Statistical Package for Social Sciences (SPSS) version 17 was used for analysis of the vaginal doses. Descriptive statistics were used to evaluate the mean dose and standard deviation of vaginal-point doses in each fraction and cumulative dose in EQD2 concepts. For one patient, the cumulative dose points in EQD2 concepts at VR and VL were evaluated and the higher one was chosen to evaluate the relationship to vaginal toxicity as maximally vaginal dose (Vmax) of each patient. For evaluation of cumulative vaginal doses and vaginal stricture, Mann-Whitney test was used to evaluate.

\section{Results}

From January 2010 to December 2011, twenty-six patients were enrolled and evaluated with the median age of 53.7 years old. Seventeen of them were stage IIB according to FIGO staging. All characteristic data is shown in Table 1.

The mean doses and standard deviations of D90 of HR-CTV, D2cc of bladder, D2cc of rectum and D2cc of sigmoid colon were $95.6 \pm 8.8 \mathrm{~Gy}, 84.5 \pm 7.7 \mathrm{~Gy}, 66.8 \pm 5.9 \mathrm{~Gy}$ and $70.8 \pm 6.3 \mathrm{~Gy}$, respectively. The mean doses to right vaginal dose point (VR), left vaginal dose point (VL) and average dose of $\operatorname{VR} / \mathrm{VL}((\mathrm{VR}+\mathrm{VL}) / 2)$ were 101.5 Gy, 98.2 Gy and 99.8 Gy in EQD2 concepts, respectively. In comparison to point-based planning, volume-based planning significantly reduced the cumulative dose in EQD2 concepts at vaginal points. All data was shown in Table 2.

At the median follow-up time of 22 months (IQ range 19 - 23 months), no grade 3 vaginal stricture was observed. Sixteen patients (61.5\%) developed to grade $1-2$ toxicity and ten patients developed grade 0 vaginal stricture. All profiles are shown in Table 3.

Table 1. Characteristic data for the group in this study.

\begin{tabular}{cc}
\hline Parameters & Data \\
\hline Age (mean: IQR) & 53.7 years: 50 - 58 years \\
Stage & 17 \\
IIB & 9 \\
IIIB & \\
Pathological reports & 22 \\
Squamous cell carcinoma & 4 \\
Adenocarcinoma & \\
Whole pelvis radiotherapy & 12 \\
With 3D-conformal RT & 14 \\
With IMRT & 55.9 days: $50.8-61.2$ days \\
Total treatment time (days) (mean: IQR)
\end{tabular}

Note: IQR = Interquartile range, IMRT = Intensity-modulated radiation therapy, 3D-conformal RT = Three-dimensional conformal radiation therapy. 
Table 2. The dose at vaginal dose points per fraction, in EQD2 and in cumulative dose.

\begin{tabular}{cccc}
\hline Parameter & Point-based planning (point A) (Gy) & Volume-based planning (Gy) & P-value \\
\hline VR dose per fraction & $8.2 \pm 1.5$ & $7 \pm 1.7$ & $<0.001$ \\
VL dose per fraction & $8.1 \pm 1.5$ & $6.8 \pm 1.7$ & $<0.001$ \\
VR dose per fraction in EQD2 & $19 \pm 5.6$ & $15.6 \pm 6.1$ & $<0.001$ \\
VL dose per fraction in EQD2 & $18.6 \pm 5.5$ & $13.7 \pm 5.8$ & $<0.001$ \\
Cumulative VR dose in EQD2 & $119.4 \pm 19.3$ & $101.5 \pm 18.4$ & $<0.001$ \\
Cumulative VL dose in EQD2 & $117.5 \pm 18.8$ & $98.2 \pm 18$ & $<0.001$ \\
Maximal vaginal-point dose in EQD2 & $121.6 \pm 19.8$ & $101.1 \pm 19.4$ & $<0.001$ \\
\hline
\end{tabular}

Table 3. Reported vaginal stricture in study group.

\begin{tabular}{cc}
\hline Parameters & Vaginal stricture $(\mathrm{N}=26)$ \\
\hline Grade 0 & $10(38.5 \%)$ \\
Grade 1 & $14(53.8 \%)$ \\
Grade 2 & $2(7.7 \%)$ \\
\hline
\end{tabular}

The incidences of vaginal stricture were not differed between the cumulative dose to vaginal dose points in EQD2 concepts of $\leq 90$ Gy versus $>90$ Gy $(P=1.000)$ and $\leq 100$ Gy versus $>100$ Gy $(P=0.815)$. All data was shown in Table 4.

\section{Discussion}

The measurement of vaginal dose has been debated for a long time. No definite data onto vaginal dose evaluation and related toxicities was reported. According to ABS recommendations, point-based evaluation still use [19]. With the new era of IGBT, many trials were performed to evaluate the correlation in volume-based concepts. Berger et al. and Fidarova et al. reported that dose volume parameter D2cc does not correlate with vaginal side effects of individual patients. The mean dose reported (D2cc) to the upper vagina which receives very high doses was 141 Gy EQD2 and no correlation could be demonstrated between D2cc dose volume parameter and the vaginal toxicity. Moreover, Rai et al. reported the evaluation of vaginal doses and toxicity in image-guided brachytherapy for cervical cancer. They found no association with the dose volume parameters and vaginal toxicity. The reasons can be due to difficulty in assessing the dose to the vagina as high variations in the contouring and geometric positioning that have been reported using both point dose values and DVH parameters for the vaginal wall [9] [20] [21].

In our study, volume-based planning significantly reduced the cumulative dose in EQD2 concepts at vaginal points. The incidence of vaginal stricture in our study was $61.5 \%$ in comparison to vaginal toxicity of $85.3 \%$ from the publication of Pötter et al. [2]. Moreover, the incidences of vaginal stricture were not differed between the cumulative dose to vaginal dose points in EQD2 concepts of $\leq 90$ Gy versus $>90$ Gy $(P=1.000)$ and $\leq 100$ Gy versus $>100$ Gy $(P=0.815)$. After treatment finished, our patients were encouraged to perform dilation. This procedure may reduce the incidence of vaginal stricture in our study. Unfortunately, Miles et al. reported that no reliable evidence to suggest that routine dilatation prevented vaginal toxicity [22].

However, this study has some limitations. The vaginal doses were evaluated according to ABS recommendations might not represent to the overall vaginal dose. The other performed to approach for vaginal dose evaluation is using additional dose points. The study by Westerveld et al. reported the vaginal dose concepts that defined the points of/in the level of Posterior-Inferior Border of Symphysis (PIBS) with $\pm 2 \mathrm{~cm}$ in supero-inferior aspects in four quadrants the vagina (3/6/9/12 o clock). At the vaginal surface at ring level measured doses were respectively 266.1 Gy (67.6 - 814.5)/225.9 Gy (61.5 - 610.5) at 3/9 o’clock, and 85.1 Gy (55.4 - 140.3)/72.0 Gy (49.1 - 108.9) at 12/6 o'clock [23]. However, identification the thickness of vagina in many directions need MRI images that could not be approached in all institutions. In the CT-based treatment, the optimally vaginal dose 
Table 4. The association between cumulatively vaginal dose and vaginal stricture.

\begin{tabular}{ccccc}
\hline Cumulative dose to vagina & $\mathrm{N}$ & Mean toxicity & Standard deviation & P-value \\
\hline$\leq 90 \mathrm{~Gy}$ & 9 & 0.667 & 0.50 & 1.000 \\
$>90 \mathrm{~Gy}$ & 17 & 0.706 & 0.686 & P-value \\
\hline Cumulative dose to vagina & $\mathrm{N}$ & Mean toxicity & Standard deviation & 0.815 \\
$\leq 100 \mathrm{~Gy}$ & 11 & 0.636 & 0.505 & 0.704 \\
$>100 \mathrm{~Gy}$ & 15 & 0.733 & & \\
\hline
\end{tabular}

measurement is under investigation.

\section{Conclusion}

In conclusion, there was no association found between calculated cumulative vaginal doses and events of vaginal stricture in this study. Future evaluation in many points of the vagina according to PIBS may reveal more information.

\section{Acknowledgements}

The author offers many thanks to the NRU-CMU in the Gynecologic Oncology Cluster, the Research Unit of Faculty of Medicine, Chiang Mai University and Nucletron for partial support. Definitely, the author conveys many thanks to our staff at the division of therapeutic radiology and oncology, faculty of medicine, Chiang Mai University, for supporting this study.

\section{Funding}

This work was supported by The National Research University Project (NRU) under Thailand's Office of the Higher Education Commission for financial support.

\section{References}

[1] Kamnerdsupaphon, P., Srisukho, S., Sumitsawan, Y., Lorvidhaya, V. and Sukthomya, V. (2008) Cancers in Northern Thailand. Biomedical Imaging and Intervention Journal, 4, e46. http://dx.doi.org/10.2349/biij.4.3.e46

[2] Pötter, R., Georg, P., Dimopoulos, J.C., Grimm, M., Berger, D., Nesvacil, N., et al. (2011) Clinical Outcome of Protocol Based Image (MRI) Guided Adaptive Brachytherapy Combined with 3D Conformal Radiotherapy with or without Chemotherapy in Patients with Locally Advanced Cervical Cancer. Radiotherapy and Oncology, 100, 116-123. http://dx.doi.org/10.1016/j.radonc.2011.07.012

[3] Beriwal, S., Kannan, N., Kim, H., Houser, C., Mogus, R., Sukumvanich, P., et al. (2011) Three-Dimensional High Dose Rate Intracavitary Image-Guided Brachytherapy for the Treatment of Cervical Cancer Using a Hybrid Magnetic Resonance Imaging/Computed Tomography Approach: Feasibility and Early Results. Clinical Oncology (The Royal College of Radiologists), 23, 685-690. http://dx.doi.org/10.1016/j.clon.2011.08.007

[4] Charra-Brunaud, C., Harter, V., Delannes, M., Haie-Meder, C., Quetin, P., Kerr, C., et al. (2012) Impact of 3D Image-Based PDR Brachytherapy on Outcome of Patients Treated for Cervix Carcinoma in France: Results of the French STIC Prospective Study. Radiotherapy and Oncology, 103, 305-313. http://dx.doi.org/10.1016/j.radonc.2012.04.007

[5] Tan, L.T., Coles, C.E., Hart, C. and Tait, E. (2009) Clinical Impact of Computed Tomography-Based Imaged-Guided Brachytherapy for Cervix Cancer Using the Tandem-Ring Applicator-The Addenbrooke's Experience. Clinical Oncology, 21, 175-182. http://dx.doi.org/10.1016/j.clon.2008.12.001

[6] Haie-Meder, C., Kramar, A., Lambin, P., Lancar, R., Scalliet, P., Bouzy, J., et al. (1994) Analysis of Complications in a Prospective Randomized Trial Comparing Two Brachytherapy Low Dose Rates in Cervical Carcinoma. International Journal of Radiation Oncology*Biology*Physics, 29, 953-960.

[7] Eifel, P.J., Levenback, C., Wharton, J.T. and Oswald, M.J. (1995) Time Course and Incidence of Late Complications in Patients Treated with Radiation Therapy for FIGO Stage IB Carcinoma of the Uterine Cervix. International Journal of Radiation Oncology*Biology*Physics, 32, 1289-300.

[8] Gondi, V., Bentzen, S.M., Sklenar, K.L., Dunn, E.F., Petereit, D.G., Tannehill, S.P., et al. (2012) Severe Late Toxici- 
ties Following Concomitant Chemoradiotherapy Compared to Radiotherapy Alone in Cervical Cancer: An Inter-Era Analysis. International Journal of Radiation Oncology*Biology*Physics, 84, 973-982.

[9] Fidarova, E.F., Berger, D., Schüssler, S., Dimopoulos, J., Kirisits, C., Georg, P., et al. (2010) Dose Volume Parameter D2cc Does Not Correlate with Vaginal Side Effects in Individual Patients with Cervical Cancer Treated within a Defined Treatment Protocol with Very High Brachytherapy Doses. Radiotherapy and Oncology, 97, 76-79. http://dx.doi.org/10.1016/j.radonc.2010.05.005

[10] Tharavichitkul, E., Chakrabandhu, S., Wanwilairat, S., Tippanya, D., Nobnop, W., Pukanhaphan, N., et al. (2013) Intermediate-Term Results of Image-Guided Brachytherapy and High-Technology External Beam Radiotherapy in Cervical Cancer: Chiang Mai University Experience. Gynecologic Oncology, 130, 81-85. http://dx.doi.org/10.1016/j.ygyno.2013.04.018

[11] Lim, K., Small Jr., W., Portelance, L., Creutzberg, C., Jürgenliemk-Schulz, I.M., Mundt, A., et al. (2011) Consensus Guidelines for Delineation of Clinical Target Volume for Intensity-Modulated Pelvic Radiotherapy for the Definitive Treatment of Cervix Cancer. International Journal of Radiation Oncology, Biology, Physics, 79, 348-355. http://dx.doi.org/10.1016/j.ijrobp.2009.10.075

[12] Taylor, A., Rockall, A.G., Reznek, R.H. and Powell, M.E. (2005) Mapping Pelvic Lymph Nodes: Guidelines for Delineation in Intensity-Modulated Radiotherapy. International Journal of Radiation Oncology, Biology, Physics, 63, 1604-1612. http://dx.doi.org/10.1016/j.ijrobp.2005.05.062

[13] Toita, T., Ohno, T., Kaneyasu, Y., Uno, T., Yoshimura, R., Kodaira, T., et al. (2010) A Consensus-Based Guideline Defining the Clinical Target Volume for Pelvic Lymph Nodes in External Beam Radiotherapy for Uterine Cervical Cancer. Japanese Journal of Clinical Oncology, 40, 456-463. http://dx.doi.org/10.1093/jico/hyp191

[14] Toita, T., Ohno, T., Kaneyasu, Y., Uno, T., Kato, T., Hatano, K., et al. (2011) A Consensus-Based Guideline Defining Clinical Target Volume for Primary Disease in External Beam Radiotherapy for Intact Uterine Cervical Cancer. Japanese Journal of Clinical Oncology, 41, 1119-1126. http://dx.doi.org/10.1093/jjco/hyr096

[15] Joiner, M. and Van der Kogel, A. (2009) Basic Clinical Radiobiology. Edward Arnold, London.

[16] Haie-Meder, C., Pötter, R., Van Limbergen, E., Briot, E., De Brabandere, M., Dimopoulos, J., et al. (2005) Recommendations from Gynaecological (GYN) GEC-ESTRO Working Group (I): Concepts and Terms in 3D Image Based 3D Treatment Planning in Cervix Cancer Brachytherapy with Emphasis on MRI Assessment of GTV and CTV. Radiotherapy and Oncology, 74, 235-245. http://dx.doi.org/10.1016/j.radonc.2004.12.015

[17] Viswanathan, A.N., Dimopoulos, J., Kirisits, C., Berger, D. and Pötter, R. (2007) Computed Tomography versus Magnetic Resonance Image-Based Contouring in Cervical Cancer Brachytherapy: Result of a Prospective Trial and Preliminary Guidelines for Standard Contours. International Journal of Radiation Oncology, Biology, Physics, 68, 491-498. http://dx.doi.org/10.1016/j.ijrobp.2006.12.021

[18] Pötter, R., Haie-Meder, C., Van Limbergen, E., Barillot, I., De Brabandere, M., Dimopoulos, J., et al. (2006) Recommendations from Gynaecological (GYN) GEC ESTRO Working Group (II): Concepts and Terms in 3D Image-Based Treatment Planning in Cervix Cancer Brachytherapy-3D Dose Volume Parameters and Aspects of 3D Image-Based Anatomy, Radiation Physics, Radiobiology. Radiotherapy and Oncology, 78, 67-77. http://dx.doi.org/10.1016/j.radonc.2005.11.014

[19] Viswanathan, A.N. and Thomadsen, B. (2012) American Brachytherapy Society Consensus Guidelines for Locally Advanced Carcinoma of the Cervix. Part I: General Principles. Brachytherapy, 11, 33-46. http://dx.doi.org/10.1016/j.brachy.2011.07.003

[20] Berger, D., Dimopoulos, J., Georg, P., Georg, D., Pötter, R. and Kirisits, C. (2007) Uncertainties in Assessment of the Vaginal Dose for Intracavitary Brachytherapy of Cervical Cancer Using a Tandem-Ring Applicator. International Journal of Radiation Oncology, Biology, Physics, 67, 1451-1459. http://dx.doi.org/10.1016/j.ijrobp.2006.11.021

[21] Rai, B., Dhanireddy, B., Patel, F.D., Kumari, R., Oinam, A.S., Simha, V., et al. (2014) Vaginal Dose, Toxicity and Sexual Outcomes in Patients of Cervical Cancer Undergoing Image Based Brachytherapy. Asian Pacific Journal of Cancer Prevention, 15, 3619-3623. http://dx.doi.org/10.7314/APJCP.2014.15.8.3619

[22] Miles, T. and Johnson, N. (2010) Vaginal Dilator Therapy for Women Receiving Pelvic Radiotherapy. Cochrane Database of Systematic Reviews, Published Online.

[23] Westerveld, H., Pötter, R., Berger, D., Dankulchai, P., Dörr, W., Sora, M.C., et al. (2013) Vaginal Dose Point Reporting in Cervical Cancer Patients Treated with Combined 2D/3D External Beam Radiotherapy and 2D/3D Brachytherapy. Radiotherapy and Oncology, 107, 99-105. http://dx.doi.org/10.1016/j.radonc.2013.04.009 
Scientific Research Publishing (SCIRP) is one of the largest Open Access journal publishers. It is currently publishing more than 200 open access, online, peer-reviewed journals covering a wide range of academic disciplines. SCIRP serves the worldwide academic communities and contributes to the progress and application of science with its publication.

Other selected journals from SCIRP are listed as below. Submit your manuscript to us via either submit@scirp.org or Online Submission Portal.
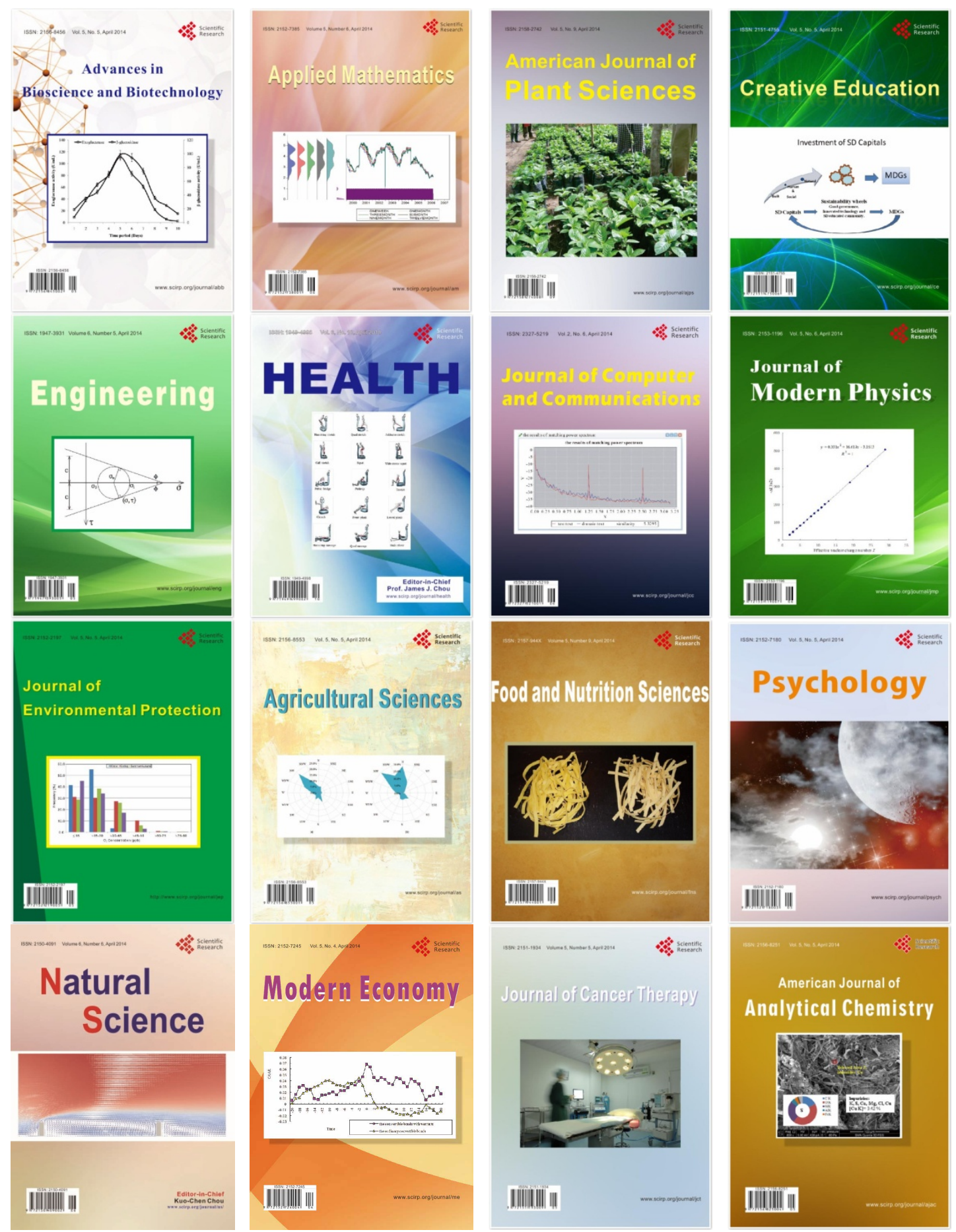